\title{
Design and fabrication of embedded micro-mirror inserts for out-of-plane coupling in PCB-level optical interconnections
}

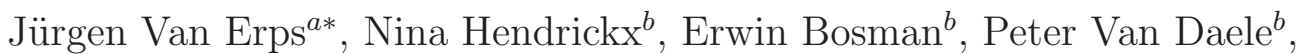 \\ Christof Debaes $^{a}$, Hugo Thienpont ${ }^{a}$ \\ ${ }^{a}$ Vrije Universiteit Brussel, Faculty of Engineering, Brussels Photonics Team (B-PHOT), \\ TONA-FirW, B-1050 Brussel, Belgium \\ ${ }^{b}$ Ghent University, Dept. of Information Technology-CMST, \\ B-9052 Zwijnaarde, Belgium
}

\begin{abstract}
Optical interconnections have gained interest over the last years, and several approaches have been presented for the integration of optics to the printed circuit board (PCB)-level. The use of a polymer optical waveguide layer appears to be the prevailing solution to route optical signals on the PCB. The most difficult issue is the efficient out-of-plane coupling of light between surface-normal optoelectronic devices (lasers and photodetectors) and PCB-integrated waveguides. The most common approach consists of using $45^{\circ}$ reflecting micro-mirrors. The micro-mirror performance significantly affects the total insertion loss of the optical interconnect system, and hence has a crucial role on the system's bit error rate (BER) characteristics.

Several technologies have been proposed for the fabrication of $45^{\circ}$ reflector micro-mirrors directly into waveguides. Alternatively, it is possible to make use of discrete coupling components which have to be inserted into cavities formed in the PCB-integrated waveguides. In this paper, we present a hybrid approach where we try to combine the advantages of integrated and discrete coupling mirrors, i.e. low coupling loss and maintenance of the planararity of the top surface of the optical layer, allowing the lamination of additional layers or the mounting of optoelectronic devices.

The micro-mirror inserts are designed through non-sequential ray tracing simulations, including a tolerance analysis, and subsequently prototyped with Deep Proton Writing (DPW). The DPW prototypes are compatible with mass fabrication at low cost in a wide variety of high-tech plastics. The DPW micro-mirror insert is metallized and inserted in a laser ablated cavity in the optical layer and in a next step covered with cladding material. Surface roughness measurements confirm the excellent quality of the mirror facet. An average mirror loss of $0.35-\mathrm{dB}$ was measured in a receiver scheme, which is the most stringent configuration. Finally, the configuration is robust, since the mirror is embedded and thus protected from environmental contamination, like dust or moisture adsorption, which makes them interesting candidates for out-of-plane coupling in high-end boards.
\end{abstract}

Keywords: coupling structures, deep proton writing, laser ablation, micro-mirror, optical interconnections, polymer waveguides, printed circuit board

\section{INTRODUCTION}

In recent years, there has been a continuing growth in the demand for data communications link capacity, which in turn has driven the need for short-reach interconnects that are capable of operating at data rates greater than $10 \mathrm{~Gb} / \mathrm{s}^{1}$ Existing interconnection technologies based on metal wiring technologies suffer from inherent disadvantages such as electromagnetic interference, size-density, power and heat dissipation issues when operating at high operating frequencies. Optical interconnects offer a promising solution to the performance bottleneck imposed by conventional electronic circuitry ${ }^{2}$ and constitute an intensive area of research both in industry and

\footnotetext{
*E-mail: Jurgen.Van.Erps@vub.ac.be, Tel.: +32 247748 71, Fax.: +32 262934 50, http://tona.vub.ac.be/Tona/
} 


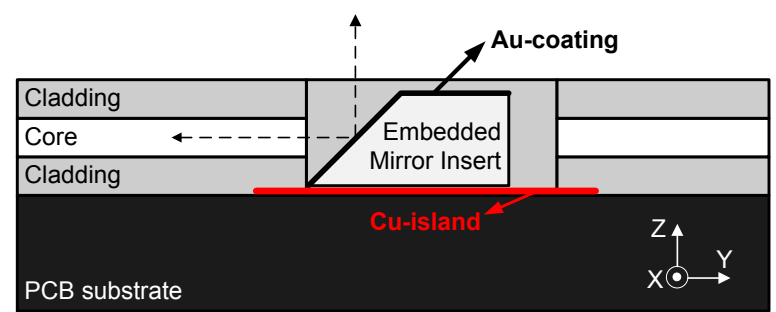

Figure 1. Operation principle of a mirror insert embedded into a cavity in the PCB: the light propagating in the optical waveguide is coupled out of plane by means of a $90^{\circ}$ deflection on the Au-coated micro-mirror or vice versa, as illustrated by the dashed arrow.

academic institutions. ${ }^{3}$ Several approaches have been presented for the integration of optics to the printed circuit board (PCB)-level. ${ }^{4}$ The use of a polymer optical layer appears to be an attractive solution, avoiding the problems associated with glass optical fiber such as high cost and minimum bending radius. ${ }^{5}$ Up to now however, no practical solution has been presented mainly because of a lack of maturity and the difficulty of coupling light efficiently in and out of the optical layer. In addition, the fabrication process has to be compatible with the existing $\mathrm{PCB}$ manufacturing and soldering processes and has to be cost-effective in order to drive $\mathrm{PCB}$ manufacturers to integrate optical interconnects into their high-end products.

The optical layer contains polymer waveguides, ${ }^{6}$ used to route high speed signals over the board, typically covering distances from a couple of centimeters up to one meter. Multimode waveguides have received particular attention owing to their relaxed alignment tolerances that allow for reduced costs for packaging and connectorization. Efficient and cost-effective coupling of light in and out of the waveguides is also being thoroughly investigated. Several different approaches have been suggested and demonstrated, including butt-coupling using fiber ribbons ${ }^{7}$ or appropriately mounted VCSEL and photodiode arrays, ${ }^{8}$ as well as out-of-plane coupling by means of grating couplers ${ }^{9}$ or the more commonly used $45^{\circ}$ micro-mirrors. ${ }^{10}$ The main advantages of the latter are their wavelength independency and high reproducibility. Different technologies can be used for the patterning of the micro-mirror directly into the waveguides, such as V-shaped diamond blade cutting, ${ }^{10}$ tilted exposure,${ }^{11}$ UV moulding ${ }^{12}$ and laser ablation. ${ }^{13}$ As an alternative to waveguide-integrated micro-mirrors, we recently proposed the use of discrete coupling structures, ${ }^{14,15}$ which can be plugged into a cavity into the optical layer. The advantage of these discrete coupling structure is that they can be inserted in a late stage of the production process, hence lowering the process compatibility requirements, and the fact that their concept can be easily extended towards multilayer coupling structures ${ }^{14}$ or curved micro-mirror for enhanced coupling efficiency. ${ }^{16}$

In this paper, we present a hybrid coupling approach in which a metallized discrete micro-mirror is embedded into the optical PCB, as illustrated in Fig. 1. ${ }^{17}$ The proposed layout is robust, since the embedded mirror is protected from environmental contamination, and the processing is relatively simple, which makes it a promising candidate for adoption by the industry. The micro-mirror inserts are designed through non-sequential ray tracing simulations, including a tolerance analysis, and subsequently prototyped with Deep Proton Writing (DPW). The DPW prototypes are compatible with mass fabrication at low cost in a wide variety of high-tech plastics. The micro-mirror components can be inserted into the optical layer using standard pick-and-place equipment, enabling a high throughput and allowing alignment accuracies of a couple of microns. We also discuss the first results of embedded micro-mirror inserts with a curved micro-mirror, aiming at collimating the beam emitted by the multimode waveguides on the $\mathrm{PCB}$ upon $90^{\circ}$ deflection.

\section{NON-SEQUENTIAL RAY TRACING SIMULATIONS}

Due to the highly multimodal character of the PCB-integrated waveguides we are considering here, ${ }^{18}$ we use non-sequential ray-tracing for the simulations of the out-of-plane coupler. We choose to use the Advanced Systems Analysis Program (ASAP) by Breault Research, since it has the possibility to include the phase and the associated polarization effects in our simulations. We do not take into account the scattering that occurs due to surface roughness (i.e. we assume perfect optical surfaces), but we do incorporate polarization effects and the Fresnel losses occurring at all the interfaces. 


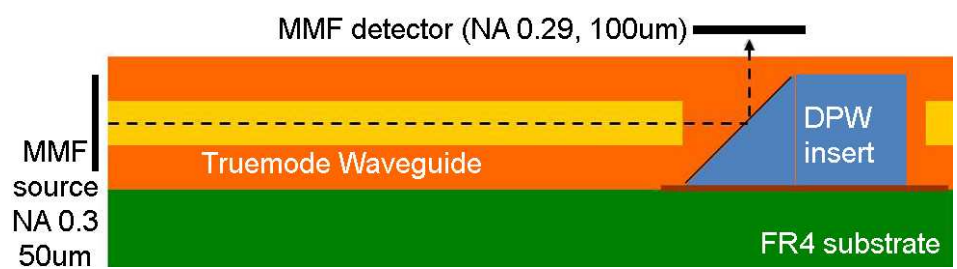

(a)

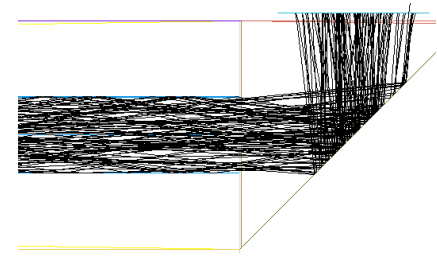

(b)

Figure 2. (a) System configuration in Rx-scheme for the non-sequential ray tracing simulations - the Truemode waveguides are 5-cm long; (b) Ray trace of the reflection at the Au-coated $45^{\circ}$ micro-mirror.

As a source, we use a completely optically filled* multimode fiber (MMF), having a core diameter of 50- $\mu m$ and a numerical aperture (NA) of 0.3. This NA corresponds exactly to the NA of the Truemode waveguides integrated on the PCB to which the light is coupled, as illustrated in Fig. 2(a). The in-plane gap between the MMF and the entrance facet of the $50 \times 50-\mu m^{2}$ Truemode waveguide is set to $5-\mu m$. We perform the simulations of the out-of-plane coupler in a receiver $(\mathrm{Rx})$ scheme, which is the most stringent one. ${ }^{15}$ In this Rx scheme, the embedded mirror insert is used to couple the light from the horizontal plane to the vertical plane, as illustrated in Fig. 2, where the light from within a PCB-integrated Truemode waveguide is coupled out of the waveguide plane towards a multimode fiber $(100-\mu m$ diameter, $\mathrm{NA}=0.29)$ as detector. For the metallized micro-mirror, the thickness of the coating is chosen to be $0.5-\mu m$, largely satisfying the requirement that the layer thickness should be large in comparison to the penetration depth of the metal used. This penetration depth $d$ can be calculated as: ${ }^{19}$

$$
d=\frac{\lambda}{4 \pi \kappa}
$$

where $\lambda$ is the wavelength in the metal and $\kappa$ represents the imaginary part of the refractive index of the metal. For gold $(\mathrm{Au})$, having a complex index of refraction of $n_{t}=0.197+5.5848 i$ at a wavelength of $850-n m,{ }^{20}$ we get a penetration depth of $d=61.5-\mathrm{nm}$. The use of Au instead of other metals is preferred, since it has the lowest absorption. Fig. 2(b) shows a ray tracing through the system, in particular the reflection at the Au-coated $45^{\circ}$ micro-mirror. At the optimal position, the maximum achievable total link coupling efficiency is $86.0 \%$ or -0.65 $d B$. This includes the coupling from the input MMF to the PCB-integrated waveguides, propagation through these 5-cm-long Truemode waveguides, reflection at the Au-coated micro-mirror and coupling towards the MMF detector.

Next to the maximum achievable optical transmission efficiency, a very important specification is the tolerance for mechanical misalignments of the detector. Especially in view of assembly and packaging, this is extremely important, since it determines the accuracy needed for the assembly or, inversely, allows one to predict the optical performance that is to be expected when the system is assembled with a certain accuracy. For example, if we want to couple the light reflected out-of-plane into a multimode fiber, what is then the allowed lateral misalignment of this fiber if we don't want the coupling efficiency to drop more than 1- $d B$ ? To simulate this, we move the detector fiber from $-100-\mu m$ to $100-\mu m$ along the $\mathrm{X}$ - and the Z-axis, with respect to the base position (i.e. the position where optimal coupling efficiency is achieved), and calculate the efficiency at each position. Fig. 3(a) shows the simulated alignment tolerance of the MMF detector for lateral misalignments (along the Xand Y-axis). The $-1-d B$ tolerance range is $\pm 29-\mu m$. In Section 5 , we will compare the experimentally measured tolerance for mechanical misalignments with the simulated values. We also simulate the effect of a detector misalignment along the Z-axis for the Truemode waveguide source. An excess loss of 1- $d B$ occurs for an 85$\mu m$ distance increase between the exit facet of the out-of-plane coupler and the detector. This is primarily a consequence of the divergence of the light beam.

\section{FABRICATION OF THE OPTICAL PCB}

Truemode Backplane ${ }^{\mathrm{TM}}$ Polymer, a highly cross-linked acrylate-based material, is used for the optical layer. This polymer material is commercially available (Exxelis Ltd., UK), shows excellent thermal and environmental

\footnotetext{
${ }^{*}$ This means that each point of the waveguide core cross-section emits an equal irradiance distribution
} 


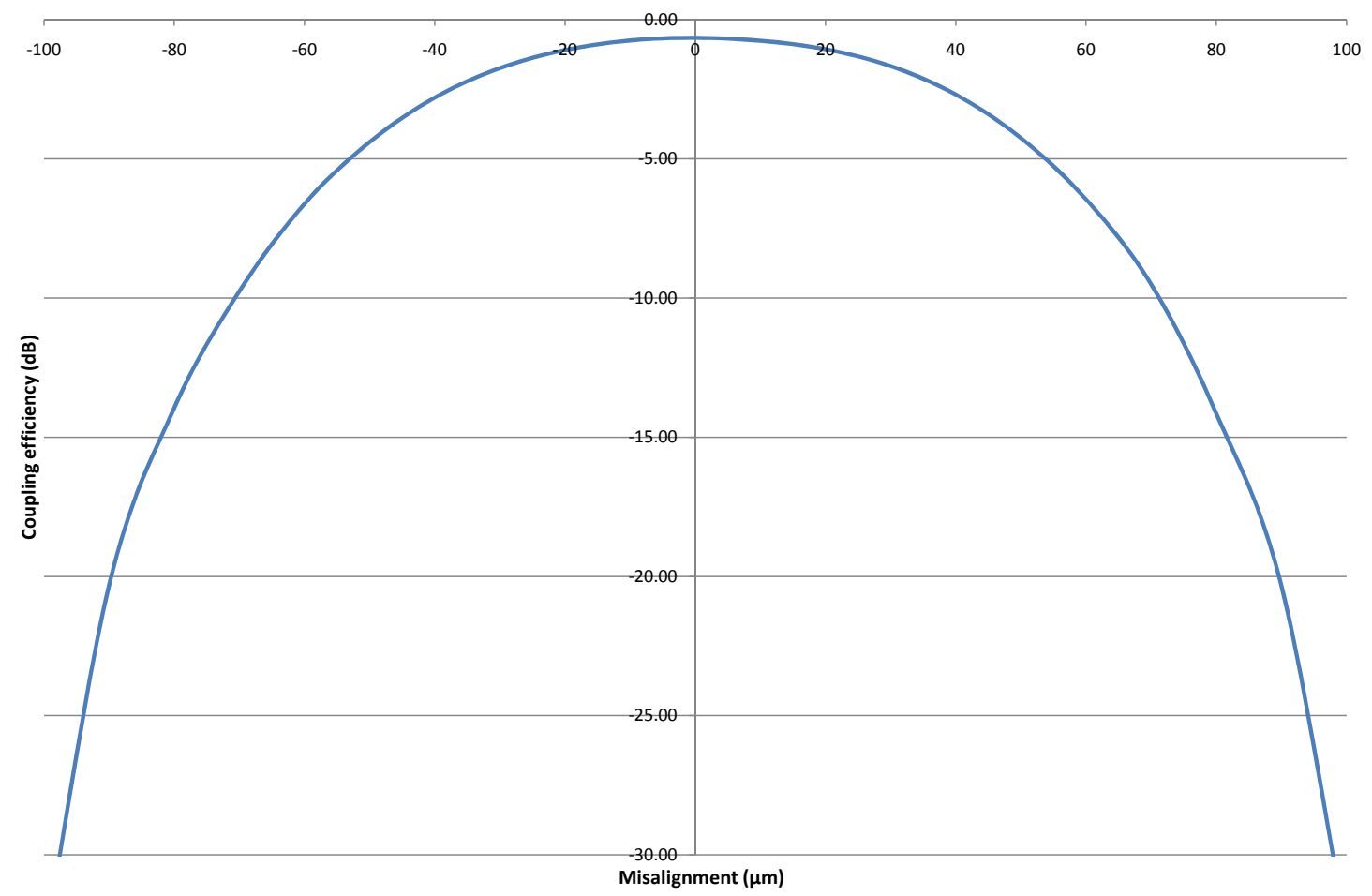

(a) Tolerance for lateral detector misalignments

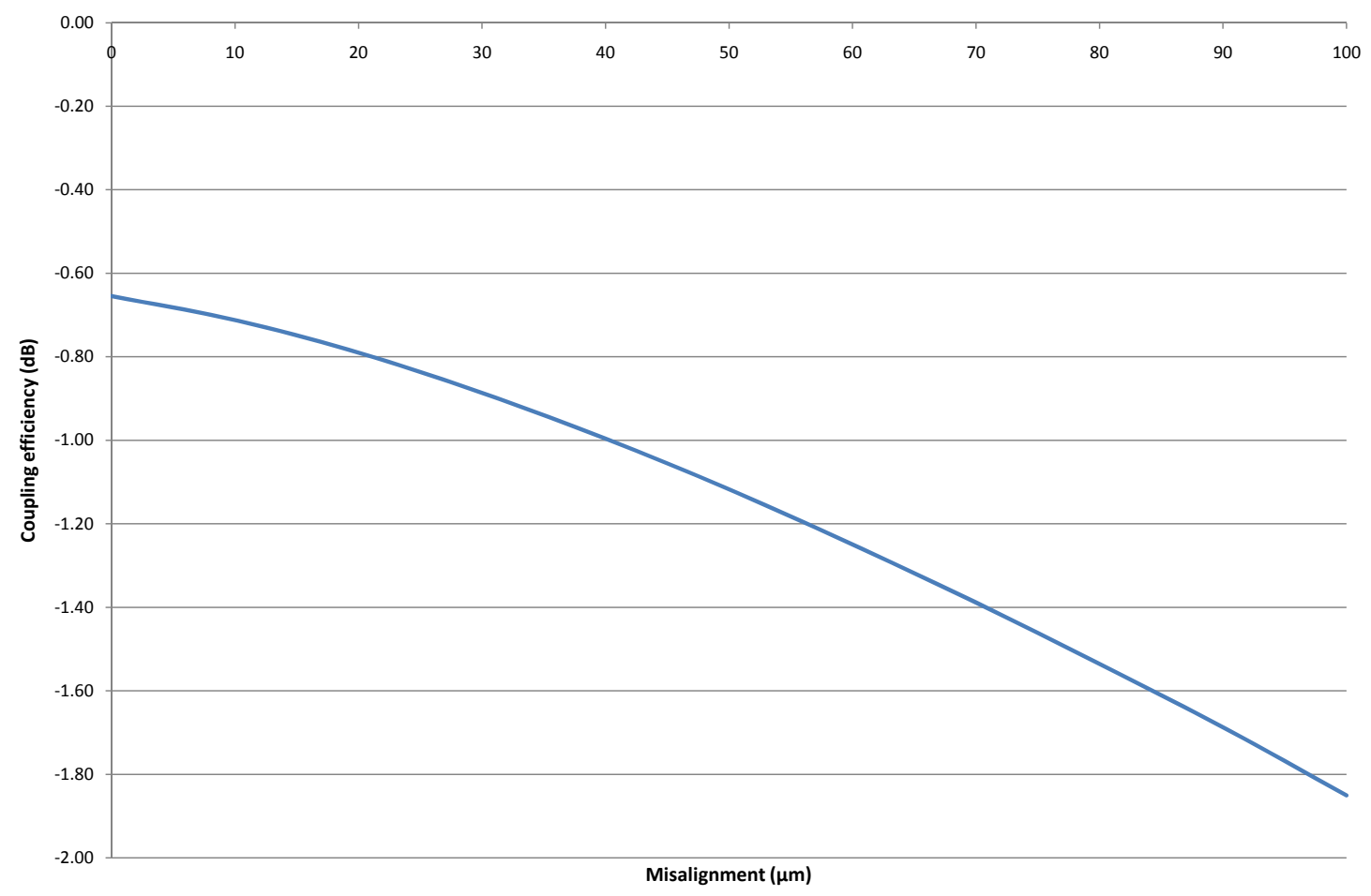

(b) Tolerance for longitudinal detector misalignments

Figure 3. Simulated alignment tolerance of the MMF detector along the X-axis (a) and along the Z-axis (b), showing a $-1-d B$ tolerance range of $\pm 29-\mu m$ and $85-\mu m$ respectively. 


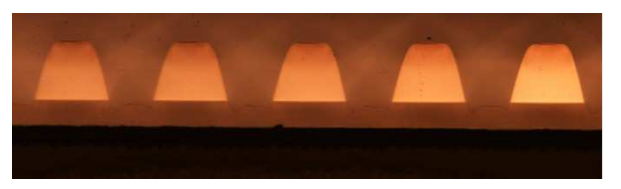

Figure 4. Polished end-facet cross-section of an array of laser ablated $50 \times 50-\mu m^{2}$ waveguides, on a pitch of $125-\mu m$.

properties and is compatible with standard PCB processing. The optical layer consists of a cladding-core-cladding stack, in which the cladding material has a slightly lower refractive index than the core material to allow light to be trapped in the core layer. Multimode optical waveguides are patterned into the core layer with use of KrF excimer laser ablation. ${ }^{21}$ The waveguides have a cross-section of about $50-\mu \mathrm{m} \times 50-\mu \mathrm{m}$ on a pitch of $125-\mu \mathrm{m}$ and have a propagation loss of $0.12-\mathrm{dB} / \mathrm{cm}$ at $850 \mathrm{~nm}$. A cross-section of an array of laser ablated waveguides is shown in Fig. 4.

To accommodate the micro-mirror insert, a micro-cavity is ablated into the optical layer by means of a KrF excimer laser. Because there is always a certain amount of tapering during the ablation, the laser beam has to be slightly tilted to obtain a vertical end-facet of the waveguides at the cavity edge. As a result, the other interface of the cavity is angled under the double tapering angle. The substrate contains a $\mathrm{Cu}$ island, as shown in Fig. 1, which is used as an ablation stop layer during the formation of the cavity. This way, the presence of a tilt of the laser ablated cavity bottom is avoided. The flatness of the cavity bottom is in correspondence with the flatness of the FR4 substrate, which is acceptable for a correct insertion of the micro-mirror component. The bottom of the ablated cavity has an average RMS surface roughness of 781- $\mathrm{nm}$ on a scan area of $0.92-\mathrm{mm} \times 1.2-\mathrm{mm}$, measured with a non-contact optical surface profiler (Wyko NT2000, Veeco). The tilt of the bottom of the cavity is $\leq 1^{\circ}$. Planarity of the bottom surface is required to preserve the $45^{\circ}$ angle of the mirror facet: angular misalignment would induce a non-perpendicular out-of-plane coupling and consequently a reduction in coupling efficiency.

\section{MICRO-MIRROR INSERT FABRICATION AND EMBEDDING INTO THE OPTICAL PCB}

For the manufacturing of the pluggable out-of-plane coupling components, we use the rapid prototyping technology of Deep Proton Writing (DPW). ${ }^{22}$ DPW consists of a patterned 8.3- $\mathrm{MeV}$ proton irradiation of $500-\mu \mathrm{m}$ thick polymer photoresist (PMMA), followed by a selective etching process to remove the exposed zones. The sample is moved according to a predefined pattern in steps of 500- $\mathrm{nm}$ with an accuracy of 50- $\mathrm{nm}$, while depositing $2.5 \times 10^{4} / \mu \mathrm{m}^{2}$ protons per step to achieve the highest quality optical surfaces. Typical proton currents for the $125-\mu \mathrm{m}$ proton beam diameter are $160-p A$, resulting in total processing times of about 3 hours (development included) for a component with dimensions of 1.0-mm $\times 0.13-\mathrm{mm} \times 0.5-\mathrm{mm}$. These dimensions can be changed if required, in view of the manageability of the insert. Fig. 5(a) shows a microscope image of the DPW-fabricated micro-mirror insert after the etching process and before the release from the 0.5-mm-thick PMMA substrate. The surface roughness of the micro-mirror facet has been measured with an optical profiler (Wyko NT2000), showing an average RMS surface roughness of 13.4- $\mathrm{nm} \pm 2.6-\mathrm{nm}$, when averaging 5 measurements of an area of $60-\mu \mathrm{m} \times 48-\mu \mathrm{m}$ at random locations. Fig. 5(b) shows a Scanning Electron Microscope (SEM) image of the prototyped $45^{\circ}$ micro-mirror insert which confirms the smooth mirror surface. The $45^{\circ}$ angle of the mirror has been checked with stylus profiler (Dektak 8, Veeco) measurements: $44.8^{\circ} \pm 0.3^{\circ}$.

It is obvious that DPW is not a mass fabrication technique as such. However, one of its assets is that once the master component has been prototyped with DPW, a metal mould can be generated from the master by applying e lectroplating. After removal of the plastic master, this metal mould can be used as a shim in a final micro-injection moulding or hot embossing step. ${ }^{23}$ This way, the component can be mass-produced at low cost in a wide variety of high-tech plastics. ${ }^{24}$

Before fixation of the micro-mirror insert in the laser ablated cavity of the optical PCB, an Au-coating is evaporated on the micro-mirror facet. The insert is attached to the $\mathrm{Cu}$ bottom surface of the laser ablated cavity using a small amount of glue, which is cured subsequently in a convection oven at $150^{\circ}$ for 5 minutes. The alignment between the mirror and the output facet of the waveguides is arranged manually by means of tweezers. However, fine-placement equipment could be used to increase the alignment accuracy and speed up the 


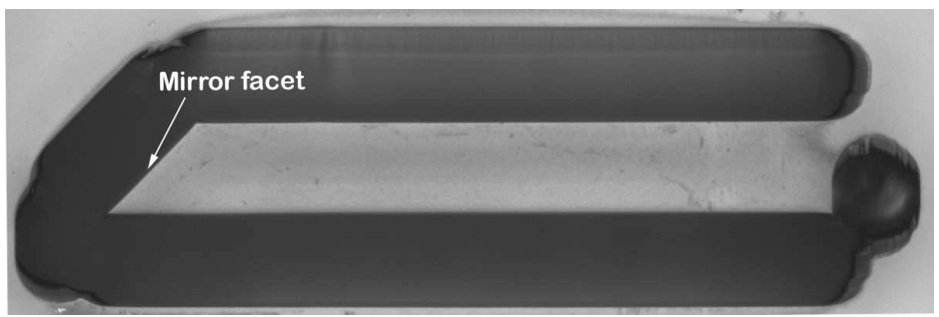

(a)

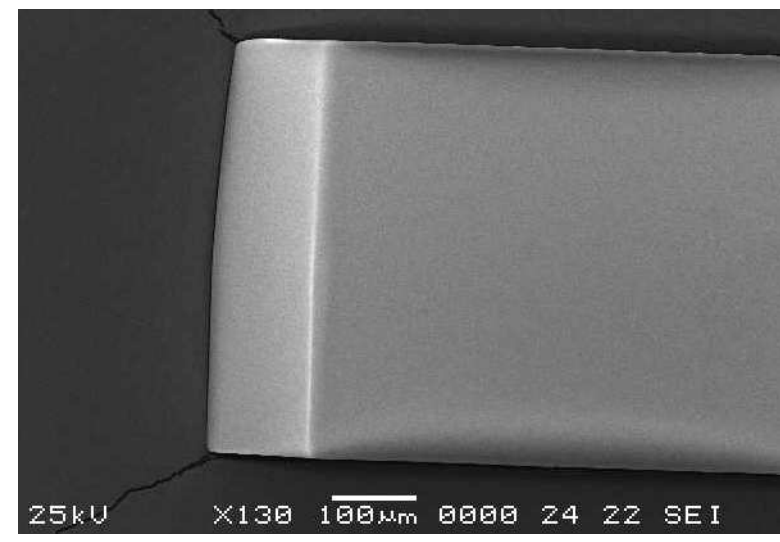

(b)

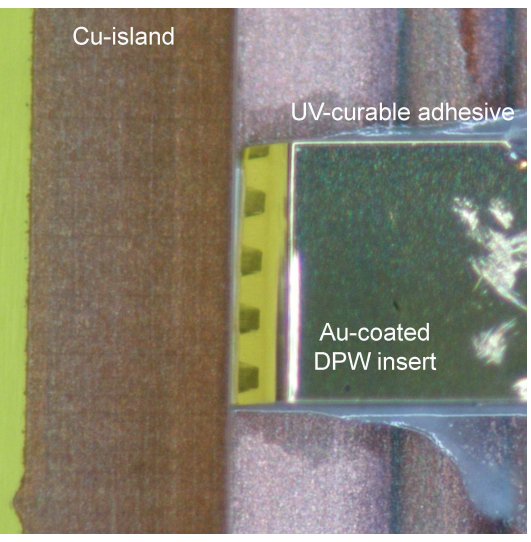

(c)

Figure 5. (a) Side view microscope image of the DPW micro-mirror insert, after the etching process before release from the PMMA substrate; (b) Top view SEM image of the DPW micro-mirror insert; (c) Top view of the metallized micro-mirror glued to the bottom of the ablated cavity, before filling it with cladding material. The reflection of the waveguide cores can be clearly observed on the mirror facet.

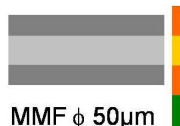

NA 0.2

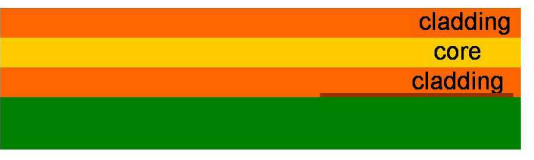

(a)

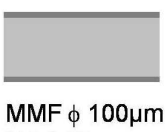

NA 0.29

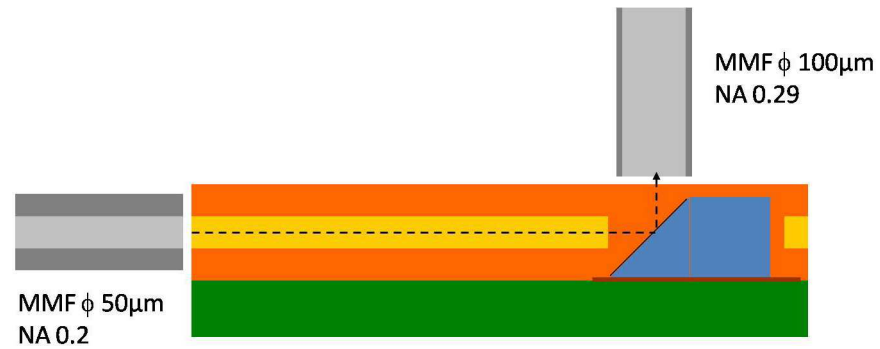

(b)

Figure 6. Experimental setup for coupling loss measurements: in-plane reference measurement (a), and measurement of the link efficiency in a receiver scheme after the embedding of the micro-mirror into the optical layer (b).

assembly process. Fig. 5(c) shows a top view of a laser ablated cavity with the DPW micro-mirror insert glued to the $\mathrm{Cu}$ bottom surface of the cavity. The reflection of the output facets of the waveguides can be clearly seen on the mirror facet. After fixation of the DPW micro-mirror insert, the cavity is filled with Truemode cladding material. 


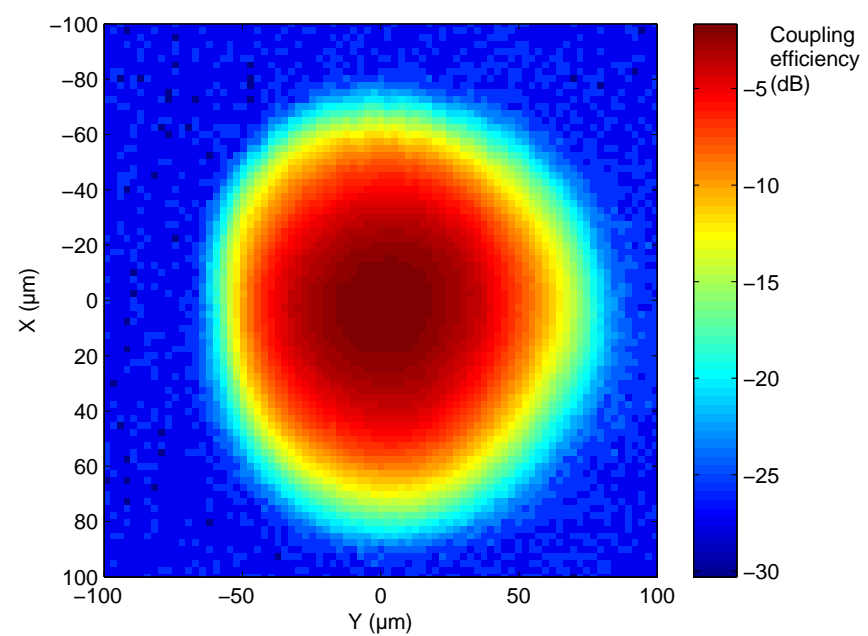

Figure 7. The alignment tolerance plot of the coupling efficiency clearly shows the circularity of the outcoupled spot. The measured -1 -dB tolerance range is $\pm 24-\mu \mathrm{m}$.

\section{OUT-OF-PLANE COUPLING EXPERIMENTS}

Before ablation of the cavity, an in-plane reference measurement is carried out on a board that contains a $\mathrm{Cu}-$ island and an array of waveguides (see Fig. 6(a)). The light is coupled in horizontally with a multimode optical fiber with a core diameter of $50-\mu \mathrm{m}$ and a numerical aperture (NA) of 0.2 . The light then propagates through the 5 - $\mathrm{cm}$ long Truemode waveguides, which have an NA of 0.3 . The optical signal that is coupled out horizontally at the output facet of the waveguides is detected with a MMF with a core diameter of 100- $\mu$ m and NA of 0.29 .

In a next step, the micro-cavity is ablated into the optical layer and the micro-mirror insert is embedded into the cavity. A loss measurement is again carried out on the board to evaluate the coupling efficiency of the embedded micro-mirror in a Rx-configuration as shown in Fig. 6(b). In-plane coupling is again performed with a MMF with a core diameter of $50-\mu \mathrm{m}$ and NA of 0.2 . The light that propagates through the Truemode waveguides is deflected over $90^{\circ}$ at the Au-coated mirror facet. The deflected light beam is detected vertically with a MMF with a core diameter of $100-\mu \mathrm{m}$ and NA of 0.29 . Comparison of the in-plane reference measurement and the out-of-plane measurement, indicates that the excess loss caused by the embedded mirror is $\leq 0.35 \mathrm{~dB}$. We also measured the tolerance for misalignments of the detector fiber. The tolerance plot of Fig. 7 shows that the $-1 \mathrm{~dB}$ tolerance range is $\pm 24-\mu \mathrm{m}$. In Fig. 8, we compare the experimentally measured tolerance for lateral misalignments along the $\mathrm{X}$-axis, with the simulation results obtained in section 2 . From this figure, it can be seen that the simulated $-1-\mathrm{dB}$ tolerance range of $\pm 29-\mu \mathrm{m}$ is slightly larger than the experimentally measured value, but overall there is a good agreement.

\section{EXTENSION TOWARDS INSERTS WITH A CURVED MICRO-MIRROR}

We have previously shown that using a curved micro-mirror rather than a standard $45^{\circ}$ mirror enables us to achieve a higher out-of-plane coupling efficiency. ${ }^{16}$ Indeed, the curvature can be chosen such that the micro-mirror incorporates some beam collimation functionality. This is especially important in view of the large divergence of the PCB-integrated multimode waveguides (NA 0.3). The design methodology consists in combining the optical simulation software ASAP and the downhill simplex numerical optimization algorithm as it is implemented in Matlab. The mirror is represented as a Bézier curve in order to limit the number of parameters to optimize. ${ }^{16}$

Fig. 9(a) shows a ray tracing through the system with the final (optimized) curved micro-mirror. The coupling efficiency at the optimal position of the embedded mirror insert with a $1 \mathrm{D}$ curved micro-mirror is $87.1 \%$ in the receiver scheme, with the same source and detector settings as described in section 2 . This turns out to be only a marginal improvement over the "standard" $45^{\circ}$ micro-mirror insert (which had a maximum coupling efficiency of $86.0 \%$ according to our simulations). The reason for this relatively small improvement compared to our previous results on curved mirror out-of-plane couplers ${ }^{16}$ is the relatively small distance the light has to travel 


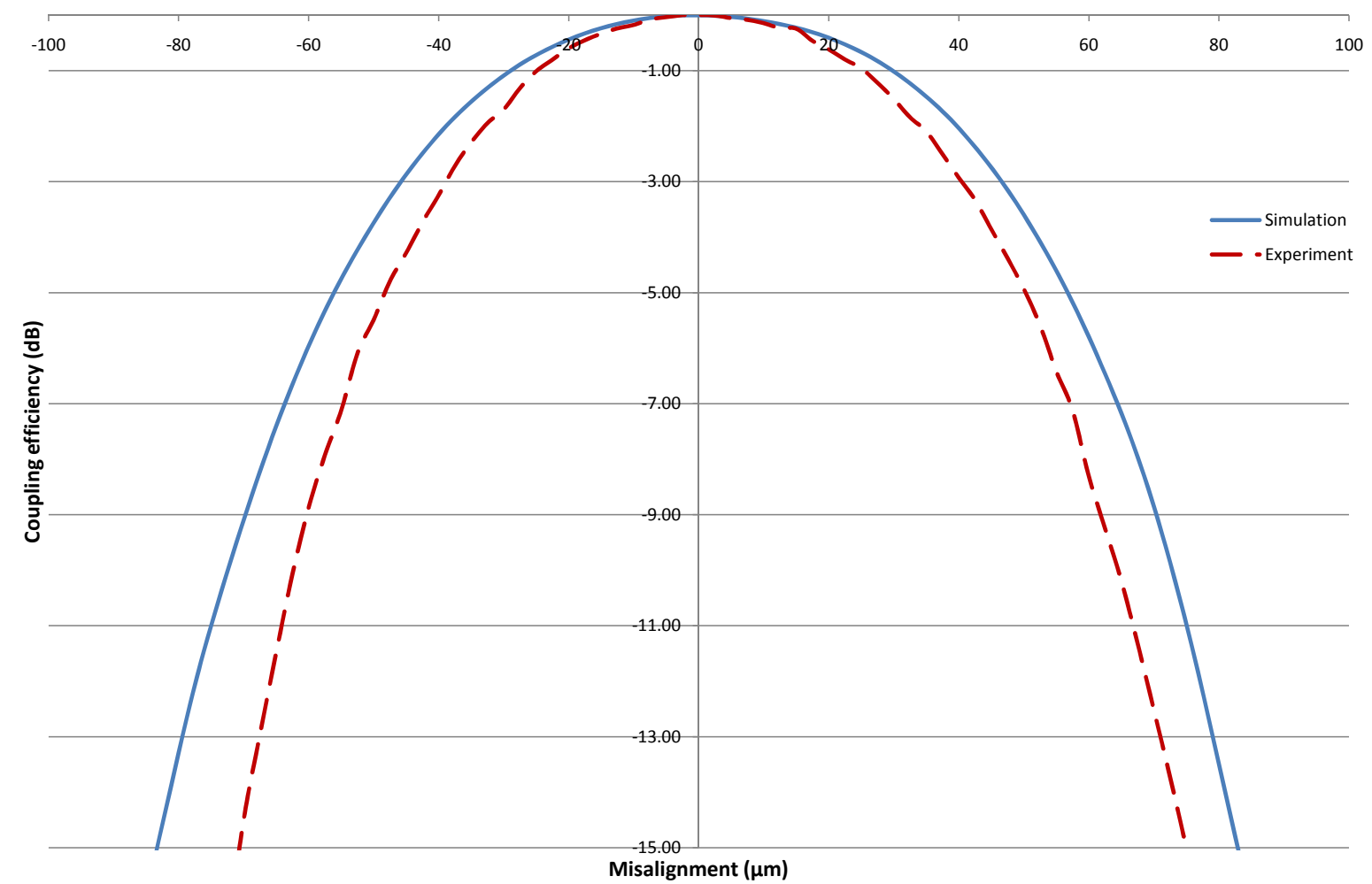

Figure 8. Simulated versus experimentally measured tolerance range for a lateral misalignment of the detector fiber along the $\mathrm{X}$-axis.

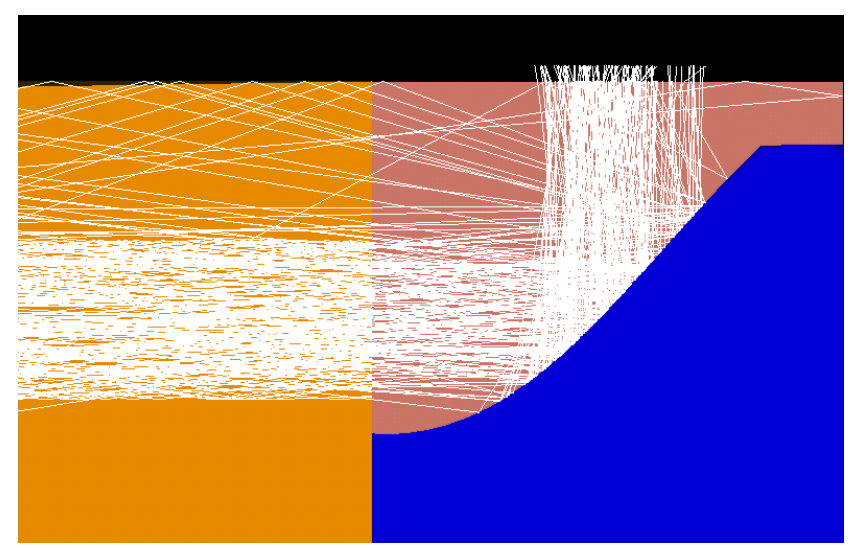

(a)

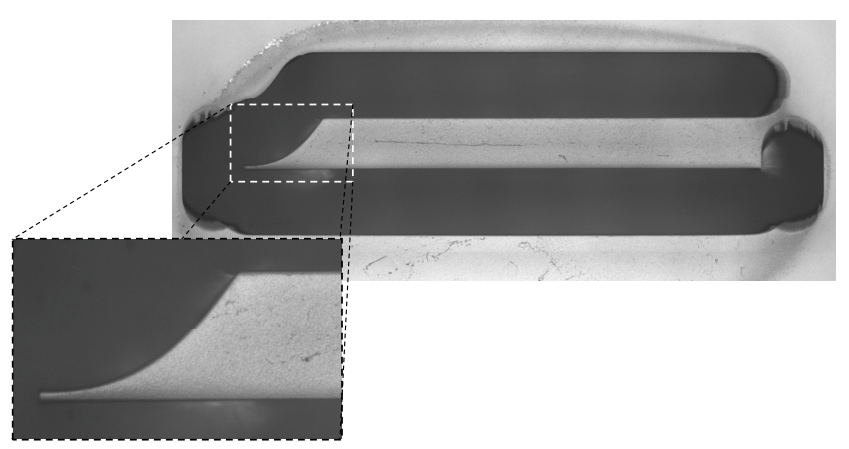

(b)

Figure 9. Design of a curved mirror insert through non-sequential ray tracing simulations (a) and DPW-fabricated prototype resulting from the design optimization (b). 
between the output facet of the board-embedded waveguide and the entrance facet of the MMF detector in this configuration. Nevertheless, because the curved micro-mirror collimates (in 1 direction) the beam emitted by the board-embedded waveguides, this leads to a slightly more relaxed longitudinal misalignment tolerance. A real advantage might be provided by a 2D-curved micro-mirror, which would collimate the out-of-plane coupled beam in 2 directions. Further simulations will be performed to evaluate this. A first DPW prototype was fabricated and is shown in Fig. 9(b). Embedding of the component and coupling efficiency measurements are currently ongoing.

\section{CONCLUSION}

We have demonstrated the hybrid integration of micro-mirror inserts, prototyped using Deep Proton Writing, into laser ablated cavities formed in optical printed circuit boards. The embedded micro-mirror inserts feature high-quality optical surfaces. An average mirror loss of only 0.35-dB was measured in a receiver scheme, which is the most stringent configuration. The prototyped micro-mirror inserts are compatible with mass fabrication at low cost in a wide variety of high-tech plastics, which makes them interesting candidates for out-of-plane coupling in high-end boards. The layout is robust, since the mirror is embedded and thus protected from outside influence, and the fabrication process is relatively simple. Use of fine-placement equipement could lead to high alignment accuracies and a high throughput. We have also shown the first realization of an insert with a curved micro-mirror. Simulations show that by using a 1D curved micro-mirror the coupling efficiency could marginally be increased by $1.1 \%$.

\section{Acknowledgment}

This work was supported in part by BELSPO-IAP, FWO, GOA, IWT-SBO, the European Network of Excellence on Micro-Optics NEMO, the Hercules and Methusalem foundations, and by the OZR of the Vrije Universiteit Brussel. The work of J. Van Erps and C. Debaes was supported by the FWO (Fund for Scientific Research Flanders) under a research fellowship.

\section{REFERENCES}

[1] A.F. Benner, P.K. Pepeljugoski and R.J. Recio, "A roadmap to 100G Ethernet at the enterprise data center," IEEE Trans. Commun. Mag., Vol. 45, No. 11, pp. 10-17, 2007.

[2] D.A.B. Miller, "Rationale and challenges for optical interconnects to electronic chips," Proc. IEEE, Vol. 88, No. 6, pp. 728-749, 2000.

[3] S. Uhlig and M. Robertsson, "Limitations to and solutions for optical loss in optical backplanes," J. Lightwave Technol., Vol. 24, No. 4, pp. 1710-1724, 2006.

[4] M.W. Haney, H. Thienpont and T. Yoshimura, "Introduction to the issue on optical interconnects," IEEE J. Select. Topics Quantum Electron., Special Issue on Optical Interconnects, Vol. 9, No. 2, pp. 347-349, 2003.

[5] N. Bamiedakis, J. Beals, I. V., R.V. Penty, I.H. White, J.V. DeGroot and T.V. Clapp, "Cost-effective multimode polymer waveguides for high-speed on-board optical interconnects," IEEE J. Sel. Topics Quant. Electron., Vol. 45, No. 4, pp. 415-424, 2009.

[6] H. Ma, A.K.-Y. Jen and L.R. Dalton, "Polymer-based optical waveguides: Materials, processing, and devices," Adv. Mater., Vol. 14, pp. 1339-1364, 2002.

[7] J. Van Erps, N. Hendrickx, M. Vervaeke, C. Debaes, P. Yan, G. Van Steenberge, P. Van Daele and H. Thienpont, "MT-compatible interface between peripheral fiber ribbons and printed circuit board-integrated optical waveguides," Proc. SPIE, Vol. 7221, 72210C, 2009.

[8] Y.-J. Chang, D. Guidotti, L. Wan, T.K. Gaylord and G.-K. Chang, "Board-level optical-to-electrical signal distribution at $10 \mathrm{~Gb} / \mathrm{s}, "$ IEEE Photon. Technol. Lett., Vol. 18, No. 17, pp. 1828-1830, 2006.

[9] N. Destouches, D. Blanc, J. Franc, S. Tonchev, N. Hendrickx, P. Van Daele and O. Parriaux, "Efficient and tolerant resonant grating coupler for multimode optical interconnections," Opt. Express, Vol. 15, pp. 16870$16879,2007$.

[10] A. Glebov, J. Roman, M.G. Lee and K. Yokouchi, "Optical interconnect modules with fully intgrated reflector mirrors," IEEE Photon. Technol. Lett., Vol. 17, No. 7, pp. 1540-1542, 2005. 
[11] F. Wang, F. Liu and A. Adibi, "45 Degree polymer micromirror integration for board-Level threedimensional optical interconnects," Opt. Express, Vol. 17, No. 13, pp. 10514-10521, 2009.

[12] X. Dou, X. Wang, H. Huang, X. Lin, D. Ding, D.Z. Pana and R.T. Chen, "Polymeric waveguides with embedded micromirrors formed by Metallic Hard Mold," Opt. Express, Vol. 18, No. 1, pp. 378-385, 2010.

[13] N. Hendrickx, J. Van Erps, G. Van Steenberge, H. Thienpont and P. Van Daele, "Laser Ablated Micromirrors for Printed Circuit Board Integrated Optical Interconnections," IEEE Photon. Technol. Lett., Vol. 19, No. 11, pp. 822-824, 2007.

[14] J. Van Erps, N. Hendrickx, C. Debaes, P. Van Daele and H. Thienpont, "Discrete out-of-plane coupling components for Printed Circuit Board-level optical interconnections," IEEE Photon. Technol. Lett., Vol. 19, No. 21, pp. 1753-1755, 2007.

[15] J. Van Erps, C. Debaes and H. Thienpont, "Design and tolerance analysis of out-of-plane coupling components for printed circuit board-level optical interconnections," IEEE J. Sel. Topics Quant. Electron., Special Issue on Enabling technologies for digital optical communication systems, accepted for publication, 2010.

[16] J. Van Erps, S. Heyvaert, C. Debaes, B. Van Giel, N. Hendrickx, P. Van Daele and H. Thienpont, "Enhanced pluggable out-of-plane coupling components for printed circuit board-level optical interconnections," Proc. SPIE, Vol. 6992, 69920S, 2008.

[17] N. Hendrickx, J. Van Erps, E. Bosman, C. Debaes, H. Thienpont and P. Van Daele, "Embedded micromirror inserts for optical printed circuit boards," IEEE Photon. Technol. Lett., Vol. 20, No. 20, pp. 1727-1729, 2008.

[18] N. Hendrickx, J. Van Erps, G. Van Steenberge, H. Thienpont and P. Van Daele, "Tolerance analysis for multilayer optical interconnections integrated on a printed circuit board," J. Lightwave Technol., Vol. 25, No. 9, pp. 2395-2401, 2007.

[19] M. Born and E. Wolf, Principles of optics (Cambridge University Press, 1999).

[20] E. Palik, Handbook of optical constants of solids (Academic Press, 1985).

[21] G. Van Steenberge, N. Hendrickx, J. Van Erps, H. Thienpont and P. Van Daele, "Laser ablation of parallel optical interconnect waveguides," IEEE Photon. Technol. Lett., Vol. 18, No. 9, pp. 1106-1108, 2006.

[22] C. Debaes, J. Van Erps, M. Vervaeke, B. Volckaerts, H. Ottevaere, V. Gomez, P. Vynck, L. Desmet, R. Krajewski, Y. Ishii, A. Hermanne and H. Thienpont,, "Deep Proton Writing: a rapid protoyping polymer microfabrication tool for micro-optical modules," New J. Phys., Vol. 8, 270, 2006.

[23] M. Heckele and W.K. Schomburg, "Review on micro molding of thermoplastic polymers," J. Micromech. Microeng., Vol. 14, pp. R1-R14, 2004.

[24] J. Van Erps, M. Wissmann, M. Guttmann, M. Hartmann, J. Mohr, C. Debaes and H. Thienpont, "Hot embossing of microoptical components prototyped through Deep Proton Writing," IEEE Photon. Technol. Lett., Vol. 20, No. 18, pp. 1539-1541, 2008. 